\title{
Addressing BACE1 flexibility to identify inhibitors for Alzheimer's Disease therapeutics
}

\author{
Sucharita D., Soumalee B. \\ Department of Microbiology, University of Calcutta \\ sbmicrobio@caluniv.ac.in
}

\begin{abstract}
Alzheimer's Disease (AD) is the most common cause of dementia that places social, psychological and financial burden worldwide. Till date, there exists no medication addressing the cause of the disease. Accordingly, there has been an ongoing urge for the development of more effective medication targeting the underlying mechanism that is responsible for the pathological condition of the disease. BACE1 being the key enzyme that leads to the formation of the amyloid- $\beta$ peptide, the prime constituent of insoluble plaques in $\mathrm{AD}$ brains, inhibition of the enzyme is a popular mechanism for therapeutics. However, flexibility of the enzyme poses a major challenge in this field. We have therefore addressed this by sampling the conformational space of BACE1 using Markov state models generated from high-dimensional time series of molecular dynamics simulations for $3.6 \mu \mathrm{s}$. Consequently, four macrostates were obtained using the PCCA+ algorithm and the most populated conformation was used to predict potent BACE1 inhibitors through virtual screening. Subsequent experimental validation was carried to identify ZINC-02119155 as a BACE1 inhibitor with $34 \%$ inhibition activity at a concentration of $2.5 \mu \mathrm{M}$.
\end{abstract}

Key words: Markov state model (MSM), clustering, RMSD, molecular docking, virtual screening.

\section{Introduction}

$\mathrm{AD}$, is a neurodegenerative disorder, the current medication for which centres around temporary reduction of symptoms and progression of the disease without aiming the underlying cause. $\mathrm{AD}$ is pathologically marked by the existence of insoluble amyloid plaques and neurofibrillary tangles in brains [1]. BACE1 ( $\beta$-Secretase ( $\beta$-site amyloid precursor protein-cleaving enzyme1) is an enzyme that cleaves the $\beta$-amyloid precursor protein, eventually generating the amyloid- $\beta$ peptide which is believed to be responsible for the amyloid cascade [2]. This enzyme therefore remains a prime target in the development of inhibitors for the treatment or prevention of the disease [3-6]. More so because BACE1 knock-out mice have been found to be normal [7].

BACE1 is known to be highly flexible and this property has made designing or identifying its inhibitors, a key challenging task in structure-based drug designing. Structural information confirms that the conformation of the domain, containing the active site, varies between closed, semi-open and open states. These states are predominantly characterised by the change in the position of the flap that covers the active site [8]. Moreover, the flap is known to be most flexible when the enzyme is free that is in the absence of any inhibitor. Several crystal structures corroborate the inhibitor-bound enzyme to adopt the closed or semiopen conformation depending upon the inhibitor type while the unbound enzyme exists as an ensemble of conformations supporting a conformational switching from open to closed form [9]. Designing inhibitors by screening using a single crystal structure of BACE1 has not been of any use for the past few decades [10]. Results of employing ensemble docking using multiple structures have not yielded, either [11]. Here, we intend to consider the high degree of conformational flexibility of BACE1 in order to virtually screen a database of commercially available chemical compounds and finally validate through experiments. For molecular simulations to capture the functional transitions of BACE1, a biologically relevant time scale which is a much longer time-scale than can be carried out for standard all-atom molecular dynamic simulations, need to be analyzed. Therefore, an efficient method of studying the conformational dynamics of BACE1 would be by constructing Markov state models (MSM) [12] from molecular dynamics simulations.

We have simulated BACE1 for a high-dimensional time series to build statistical models and select the conformation(s) with the largest population. A map of the conformational space explored by BACE1 was obtained by clustering using the RMSD metric. Further clustering yielded differently populated macrostates. Molecular docking was carried out using representative structure from the most populated state to predict potent 
BACE1 inhibitors eventually validated through experiment.

\section{Results and Discussions}

\subsection{Molecular dynamics simulation}

Four crystal structures, two in the semi-open form (PDB ID: 4H3G, 4DJX) and two in the closed form (PDB ID: 2QMG, 3CIC) were selected on the basis of the resolution of the structures and the completeness of the structural information. Molecular dynamics simulation of $100 \mathrm{~ns}$ was carried out for all the four structures using GROMACS 4.5.5 [13]. From each of these, two structures were randomly chosen for further simulation of $100 \mathrm{~ns}$. All the simulations were replicated using 3 seeds resulting in a time series of $3.6 \mu \mathrm{s}$. RMSD plots of 36 trajectories were checked for convergence before being used further to identify the most populated, long lived, kinetically stable state of BACE1.

\subsection{Markov state model generation}

The trajectories for closed and semi-open form of BACE1 amounting to $3.6 \mu$ s consisted of structures that substantially differed in their flap conformation. Hence we have used the flap RMSD to build the Markov State model using the MSMBuilder 2.8 [14]. Using the kcenters algorithm, we have clustered the conformations into 500 microstates. The micro state model was validated using the graph of relaxation time scales and lagtime. As seen in Figure 1, the implied time scales show three distinct slow timescales that are relatively flat at a lagtime of 7 time-steps. Hence, the Markov state model was built with a time-step of 7 .

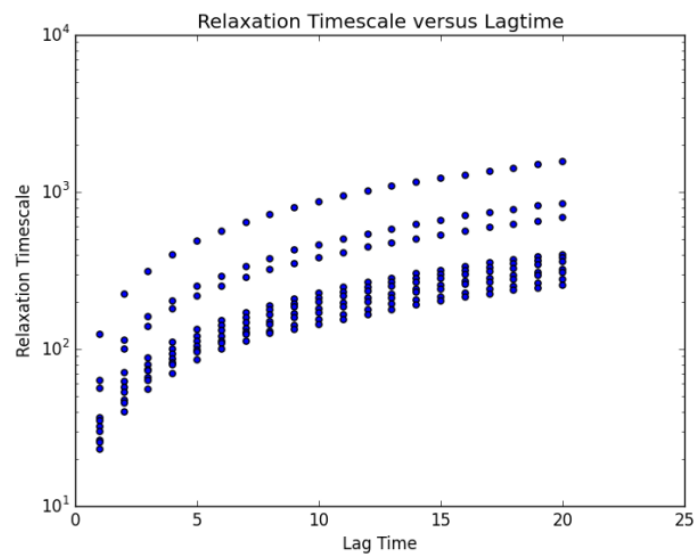

Fig. 1. Relaxation timescale of the flap in the BACE1 active-site domain.

\subsection{Selection of the most populated BACE1 conformation}

A microstate model with a short lagtime was constructed by the robust Perron Clustering Analysis (PCCA+) algorithm to create macrostates that are longlived, or metastable. This is so, since at long lagtimes, states become less and less metastable. The algorithm performs a spectral clustering based upon the eigenvalues of the transition matrix. Eigen values near to one correspond to slow transitions and can be separated from faster transitions using PCCA+. Since three slow processes were observed in the MSM, the generated microstates were clustered into 4 metastable states (Fig. 2) primarily varying in the flap orientation. The most populated $(76 \%)$ metastable state was used for virtual screening.

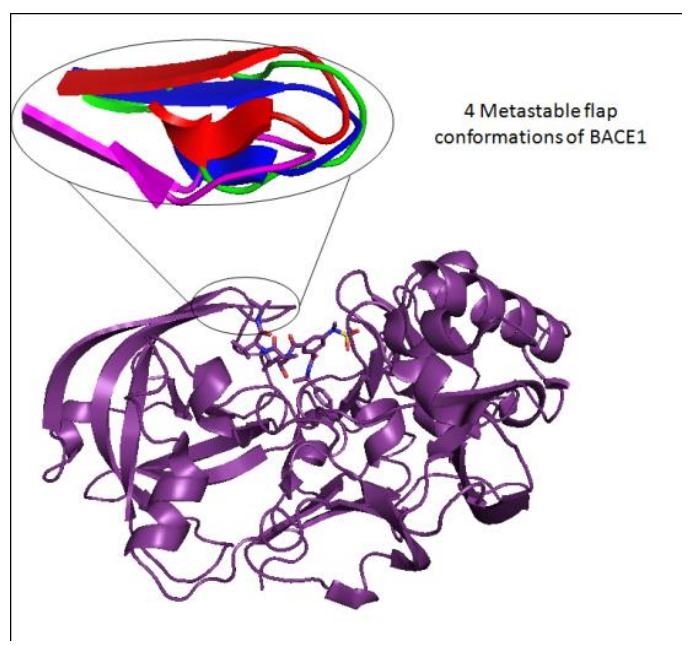

Fig. 2. Inhibitor bound BACE1 where the inhibitor is shown as sticks. Inset: Position of the flap in the four metastable states.

\subsection{Dataset generation for virtual screening}

The number of compounds present in the natural product library of the ZINC database was reduced to a sizeable number by a preliminary docking study using the closed BACE1 crystal structure (PDB ID: 2P4J). Hits having a FlexX Score $<-30 \mathrm{kcal} / \mathrm{mol}$ were considered for the subsequent virtual screening [15].

\subsection{Virtual screening}

75 compounds obtained from the preliminary docking study were virtually screened using the representative structure (the most populated metastable structure) obtained from MSM. Compounds selected on the basis of FlexX Score $<-35 \mathrm{kcal} / \mathrm{mol}$ were predicted as active BACE1 inhibitors.

\subsection{Experimental validation}

ZINC-02119155, one of the hits obtained by virtual screening was assayed for BACE1 inhibitory activity using the BACE1 assay kit. The assay is based on a convenient method of fluorescence resonance energy transfer (FRET). The substrate bears a donor and a quencher group with donor excitation at $320 \mathrm{~nm}$ and emission at $405 \mathrm{~nm}$. On cleavage by the enzyme the quencher group gets separated and fluorescence signal enhancement is observed which is used for calculating the percentage inhibition. Decrease in fluorescence signal in the presence of $2.5 \mu \mathrm{M}$ of the compound corresponded to $34 \%$ BACE1 inhibition. 


\section{Acknowledgments}

The authors acknowledge the Centre for High Performance Computing (CHPC) for Modern Biology, at Ballygunge Science College, University of Calcutta for providing the computational resources. The authors are also thankful for the UPE PhaseII funding utilized in the experimental validation. $\mathrm{SD}$ is grateful to University of Calcutta for her fellowship.

\section{References}

1. Selkoe D.J. Neuron. 1991. V. 6. P. 487-498.

2. Karran E., Mercken M., De Strooper B. Nat. Rev. Drug Discov. 2011. V. 10. P. 698-712.

3. Citron M. J. Neurosci. Res. 2002. V. 70. P. 373379.

4. Cumming J.N., Smith E.M., Wang L., Misiaszek J., Durkin J., Pan J., et al. Bioorganic Med. Chem. Lett. 2012. V. 22. P. 2444-2449.

5. Ghosh A.K., Osswald H.L. Chem. Soc. Rev. 2014. V. 43. P. 6765-6813.

6. Chakraborty S., Bandyopadhyay J., Chakraborty S., Basu S. Eur. J. Med. Chem. 2016. V. 121. P. 810-822.

7. Luo Y., Bolon B., Kahn S., et al. Nat. Neurosci. 2001. V. 4. P. 231-232. doi: 10.1038/85059.

8. XuY., Li M.J., Greenblatt H., Chen W., Paz A., Dym O., Peleg Y., Chen T., Shen X., He J., Jiang H., Silman I., Sussman J.L. Acta Crystallogr. D Biol. Crystallogr. 2012. V. 68. P. 13-25.

9. Chakraborty S., Basu S. Biophys. Chem. 2015. V. 202. P. 1-12.

10. Ghosh A.K., Bilcer G., Harwood C., Kawahama R., Shin D., Hussain K.A., et al. J. Med. Chem. 2001. V. 44. P. 2865-2868.

11. Limongelli V., Marinelli L., Cosconati S., Braun H. A., Schmidt B., Novellino E. Chem. Med. Chem. 2007. V. 2. P. 667-678.

12. Chodera J.D., Noé F. Current Opinion in Structural Biology. 2014. V. 25. P. 135-144.

13. Berendsen H.J.C., Spoel D.V.D., Drunen R.V. GROMACS: A message-passing parallel molecular dynamics implementation. Comp. Phys. Comm. 1995. V. 91. P. 43-56.

14. Harrigan M.P., Sultan M.M., Hernández C.X., Husic B.E., Eastman P., Schwantes C.R., Beauchamp K.A., McGibbon R.T., Pande V.S. MSMBuilder: Statistical Models for Biomolecular Dynamics. Biophysical Journal.2017. V. 112. P. 10-15.

15. Rarey M., Kramer B., Lengauer T., Klebe G. A fast flexible docking method using an incremental construction algorithm. J. Mol. Biol. 1996. V. 261. P. 470-89. 